\title{
Renin-angiotensin system and SARS-CoV-2 interaction: underlying mechanisms and potential clinical implications
}

\author{
Jaroslav Hrenak ${ }^{1,2, *}$, Stefan Zorad ${ }^{3}$ and Fedor Simko ${ }^{2,3,4, *}$ \\ ${ }^{1}$ Department of Cardiovascular Surgery, Inselspital - University Hospital of Bern, Bern, Switzerland \\ ${ }^{2}$ Institute of Pathophysiology, Faculty of Medicine, Comenius University, Bratislava, Slovakia \\ ${ }^{3}$ Institute of Experimental Endocrinology, Biomedical Research Center, Slovak Academy of Sciences, Bratislava, Slovakia \\ ${ }^{4} 3 \mathrm{rd}$ Department of Internal Medicine, Faculty of Medicine, Comenius University, Bratislava, Slovakia
}

\begin{abstract}
Renin-angiotensin system (RAS) inhibition supposedly increases the expression of angiotensin converting enzyme 2, serving as a binding site for SARS-CoV-2. Concerns arose regarding therapy with RAS inhibition during the COVID-19 pandemic. However, the pharmacological restraining the classical RAS axis might be beneficial due to the reduction of deleterious effects of angiotensin II and enhancement of the anti-inflammatory angiotensin 1-7 pathway. Unless large controlled studies are performed, RAS inhibition remains the cornerstone therapy in populations with cardiovascular disorders.
\end{abstract}

Key words: SARS-CoV-2 - COVID-19 - Angiotensin II - ACE 2 - ACE-inhibitor - AT1R blocker

Members of the Coronaviride family, including SARSCoV-2, bind by their Spike (S) proteins to a cell membranebound peptidase angiotensin converting enzyme 2 (ACE2), predominantly expressed in the heart, lungs and kidney (Perico et al. 2020). Treatment with ACE inhibitors or angiotensin II type 1 receptor (AT1R) blockers seems to be associated with the upregulation of ACE2, potentially accelerating the internalization of SARS-CoV-2 into target cells and sensitising patients to the virus infection. This has raised concerns regarding treatment with ACE inhibitors or AT1R blockers during the COVID-19 pandemic (Fang et al. 2020; Diaz 2020).

However, being aware of the existence of both harmful and protective cascades within the renin-angiotensin system (RAS), we suggest that the other side of the coin should also be taken into account. SARS-CoV-2 infected patients have elevated plasma levels of angiotensin II (Ang II) (Liu et al. 2020) and it has been found that the SARS-CoV-2 may downregulate ACE2 receptors (Verdecchia et al 2020). Reduced ACE2 expression may attenuate

\footnotetext{
* These authors contributed equally.

Correspondence to: Fedor Simko, Institute of Pathophysiology, Faculty of Medicine, Comenius University, Sasinkova 4, 81372 Bratislava, Slovakia

E-mail: fedor.simko@fmed.uniba.sk
}

the anti-inflammatory action of the ACE2/angiotensin (17)/Mas-receptor pathway and simultaneously potentiate the AT1R-mediated deleterious effects of abundant Ang II, in terms of exaggerated inflammation, increased capillary permeability, leading to acute lung injury and respiratory distress syndrome (Gurwitz 2020).

Accordingly, the following therapeutic targets are emerging: 1) inhibition of Ang II formation or effects; 2) stimulation of ACE2/Ang (1-7) antiinflammatory pathway; 3 ) blocking the entrance of SARS-CoV-2 into the cells. AT1R blockers or ACE inhibitors can presumably meet all three conditions. They turn down formation or effect of Ang II, alleviating its vasoconstrictive, proproliferative and proinflammatory actions, and simultaneously stimulate ACE2-mediated formation of the protective antiinflammatory metabolite angiotensin (1-7). In addition, accumulating Ang II (in the case of AT1R blockers) or Ang I (by ACE inhibitors) representing the natural agonists of ACE2, supposedly induce structural alterations in this membrane-bound peptidase, which might decrease its affinity to SARS-CoV-2 and inhibit the internalization of the virus into target cells (Perico et al. 2020).

At present, relevant clinical studies supporting or refusing the beneficial or harmful effect of RAS inhibitors in COVID-19 patients are lacking. On the other hand, the considerations about the potential replacement of ACE in- 
hibitors or AT1R blockers by other drugs (Fang et al. 2020) are not justified (Hanff et al. 2020) and may even be harmful for several following reasons:

- Patients with hypertension, coronary artery disease, heart failure, renal pathologies or diabetes mellitus have activated RAS and there is sufficient evidence that this population benefits from Ang II or ACE inhibition.

- Therapy with ACE inhibitors or AT1R blockers is gradually titrated to recommended doses. The withdrawal of these drugs may induce a rebound phenomenon, blood pressure rise, heart failure decompensation or atherosclerotic plaque instability.

- In cardiovascular disease patients with neurohumoral activation there is virtually no adequate replacement therapy, especially in the elderly population with severe cardiovascular pathologies and a saturated therapeutic schedule.

Unless large controlled studies are performed, RAS inhibition remains the cornerstone therapy in populations with cardiovascular disorders even during COVID-19 pandemy.

Acknowledgements. This work was supported by Scientific Grant Agency VEGA (No. 1/0035/19 and No. 2/0112/19).

Conflict of interest. No conflicts of interest are declared.

\section{References}

Diaz JH (2020): Hypothesis: angiotensin-converting enzyme inhibitors and angiotensin receptor blockers may increase the risk of severe COVID-19. J. Travel. Med. 27, taaa045 https://doi.org/10.1093/jtm/taaa041

Fang L, Karakiulakis G, Roth M (2020): Are patients with hypertension and diabetes mellitus at increased risk for COVID-19 infection? Lancet Respir. Med. 8, e24 https://doi.org/10.1016/S2213-2600(20)30116-8

Gurwitz D (2020): Angiotensin receptor blockers as tentative SARS-CoV-2 therapeutics. Drug. Dev. Res. (online ahead of print) https://doi.org/10.1002/ddr.21656

Hanff TC, Harhay MO, Brown TS, Cohen JB, Mohareb AM (2020): Is there an association between COVID-19 mortality and the renin-angiotensin system - a call for epidemiologic investigations. Clin. Infect. Dis. (online ahead of print) https://doi.org/10.1093/cid/ciaa329

Imai Y, Kuba K, Penninger JM (2008): Lessons from SARS: A new potential therapy for acute respiratory distress syndrome (ARDS) with angiotensin converting enzyme 2 (ACE2). Masui $\mathbf{5 7}, 302-310$

Liu Y, Yang Y, Zhang C, Huang F, Wang F, Yuan J, Wang Z, Li J, Feng C, Zhang Z, et al. (2020): Clinical and biochemical indexes from 2019-nCoV infected patients linked to viral loads and lung injury. Sci. China Life Sci. 63, 364-374 https://doi.org/10.1007/s11427-020-1643-8

Perico L, Benigni A, Remuzzi G (2020): Should COVID-19 concern nephrologists? Why and to what extent? The emerging impasse of angiotensin blockade. Nephron 144, 213-221 https://doi.org/10.1159/000507305

Verdecchia P, Cavallini C, Spanevello A, Angelib F (2020): The pivotal link between ACE2 deficiency and SARS-CoV-2 infection. Eur. J. Intern. Med. (online ahead of print) https://doi.org/10.1016/j.ejim.2020.04.037

Received: May 21, 2020

Final version accepted: May 26, 2020 